\title{
A Minimally Invasive Sinus Augmentation Technique using a Novel Bone Graft Delivery System
}

\author{
Ziv Mazor, Andreas loannou, Narayan Venkataraman, George Kotsakis
}

\begin{abstract}
A variety of surgical techniques and materials have been developed to increase the vertical bone height in the posterior maxilla. Minimally invasive crestal approaches for the elevation of the floor of the sinus using a crestal approach have been recently reported to minimize adverse events associated with the use of the osteotome technique for indirect sinus lift. The aim of this study is to report a surgical improvisation that minimizes risks associated with sinus floor elevation using a crestal approach and simplifies the clinical procedure. The technique is based on the use of a viscoelastic calcium phosphosilicate alloplastic putty (CPS putty) bone substitute that can be delivered utilizing a unique cartridge delivery system.

CPS putty not only acts as a 'protective cushion' thanks to its physical properties, but also provides hydraulic pressure to lift the Schneiderian membrane when used in conjunction with the cartridge system. This approach minimizes risks of benign paroxysmal positional vertigo or mechanical perforations of the Schneiderian membrane associated with the traditional osteotome technique.
\end{abstract}

Keywords: Indirect sinus lift, Minimally invasive sinus augmentation, Putty, Alloplastic.

How to cite this article: Mazor Z, Ioannou A, Venkataraman N, Kotsakis G. A Minimally Invasive Sinus Augmentation Technique using a Novel Bone Graft Delivery System. Int J Oral Implantol Clin Res 2013;4(2):78-82.

Source of support: Nil

Conflict of interest: None declared

\section{INTRODUCTION}

Adequate bone volume is essential for placement of implants in edentulous arches. This helps in supporting and stabilizing the body of the implant. Severe atrophy of maxillary ridges is a result of continuous pneumatization of the sinuses. Maxillary sinus augmentation surgery is frequently employed to provide adequate vertical bony dimensions for the placement of an implant.

A variety of surgical techniques and materials have been developed to increase the vertical bone height in the posterior maxilla. The aim is to increase the quantity of available bone in the area, by placement of bone-graft material in the space created following the elevation of the maxillary sinus membrane. This can be achieved either by a direct sinus lift procedure using a lateral approach and an indirect sinus lift procedure through a crestal approach, introduced by Summers in $1994{ }^{1}$
The indirect sinus elevation technique is less invasive, less time consuming with decreased postoperative patient morbidity and discomfort. Lack of direct visualization of the membrane and the use of osteotomes may lead to a risk of membrane perforation as high as $26 \%{ }^{2}$ The limit of bone volume gained with the Summers' osteotome technique is approximately up to $5 \mathrm{~mm} .^{3}$

To make the indirect sinus lift more predictable, atraumatic modifications to the Summers' technique have been developed. One such improvisation is the minimally invasive antral balloon elevation (MIAMBE). ${ }^{4}$ In this technique a transalveolar approach is utilized and the implant osteotomy is prepared 1 to $2 \mathrm{~mm}$ below the floor of the antrum. This surgical approach includes causing a small fracture in the antral floor and slowly elevating the sinus membrane with the aid of hydraulic pressure utilizing a balloon that inflates and 'pushes' the Schneiderian membrane. The gap present between the initial position of the sinus floor and the elevated membrane is filled with graft materials and an implant is placed.

In another technique, novel atraumatic drills and reamers, that can rotate in proximity to the sinus membrane and not perforate, have been utilized to make the use of osteotomes redundant. In this technique an atraumatic drill is advanced to the floor of the sinus and then a reamer is employed to drill any bone left at the floor of the sinus and elevate the membrane. Following slight elevation of the membrane with the reamer, a carrier is used to deliver bone graft through the osteotomy and further advance the membrane. ${ }^{5}$

Several bone grafting materials have been used in sinus lift procedures including autogenous bone, freeze dried bone, demineralized freeze dried bone, xenogeneic bone and alloplastic bone substitutes. ${ }^{6,7}$ Bone substitutes displaying a putty consistency are becoming more popular for bone grafting procedures. ${ }^{8,9}$ The handling characteristics of putty bone substitutes have expanded the available treatment options for bone grafting in narrow spaces and their physical properties can be exploited to increase the safety and predictability of sinus lift procedures.

In this improvisation a viscoelastic calcium phosphosilicate alloplastic putty (CPS putty) available in a unique cartridge delivery system is utilized. CPS putty is a completely synthetic graft substitute that is approved for 
bone repair and regeneration in both dental and orthopedic osseous defects. It is a premixed composite of $70 \%$ calcium phosphosilicate particulate and $30 \%$ synthetic absorbable binder. Bioactivity of CPS results from the chemical release of ionic dissolution products: silicon, sodium, calcium and phosphate and has shown to stimulate multiple generations of undifferentiated cells into osteoblasts. ${ }^{10}$ CPS has been successfully used in various osseous defects with no reported adverse events. ${ }^{9,11}$

CPS putty not only acts as a 'protective cushion' but also provides hydraulic pressure to lift the Schneiderian membrane. This approach minimizes risks of benign paroxysmal positional vertigo or mechanical perforations of the Schneiderian membrane associated with the traditional osteotome technique. In the first case example a modification of the MIAMBE technique with the use of CPS putty instead of and inflatable balloon will be presented. In the second case example, a series of atraumatic drills will be utilized in conjunction with CPS putty to perform an indirect sinus lift without the use of osteotomes.

\section{Surgical Procedure Illustration}

The technique illustrated aims to describe a modification of MIAMBE technique that employs hydraulic pressure for sinus membrane elevation. This improvization is made possible by the unique consistency and delivery mechanism of the CPS putty graft. A transalveolar sinus floor elevation (TSFE) technique is utilized and the osteotomy site is prepared to the size of the final implant diameter and stopped 0.5 to $1 \mathrm{~mm}$ short of the sinus floor (Fig. 1).

A small quantity ( $\sim 25 \mathrm{cc}$ ) of the putty graft is inserted in the implant bed to function as a 'cushion' thus preventing perforation of the membrane before the osteotome is used to tap firmly and produce a green-stick fracture (Fig. 2A).

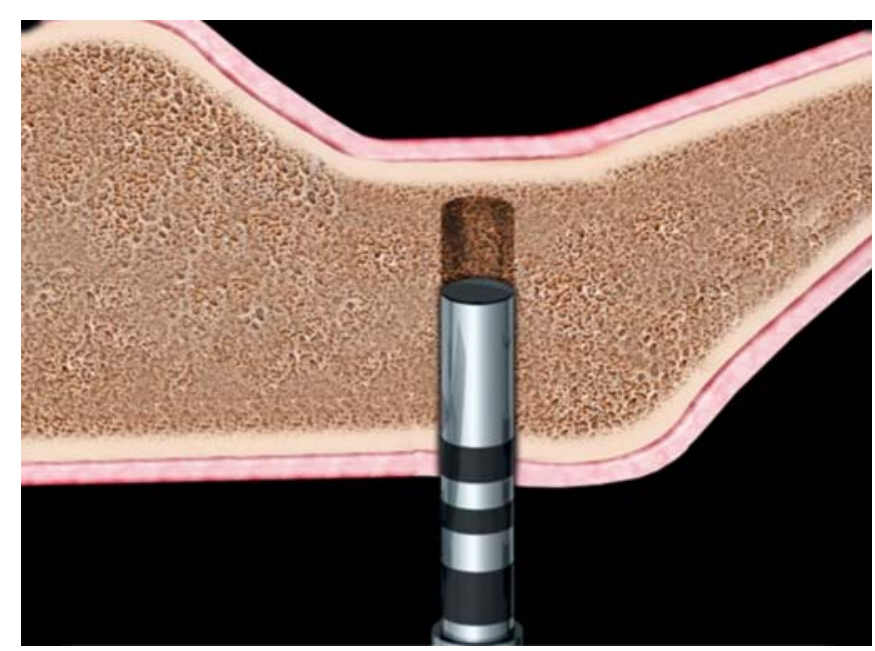

Fig. 1: In contrast to the original osteotome technique, before the in-fracture of the sinus floor with the osteotome, a small quantity of CPS putty is inserted in the osteotomy to function as a protective ‘cushion' during percussion
A putty cartridge is snapped into the dispensing gun (Fig. 2B) and the bent cannula of the cartridge is placed in the osteotomy site. The width of the cannula is narrow enough to allow it to be inserted into the osteotomy following the use of a $2.0 \mathrm{~mm}$ pilot drill. While applying pressure against the fractured bone, CPS putty is injected
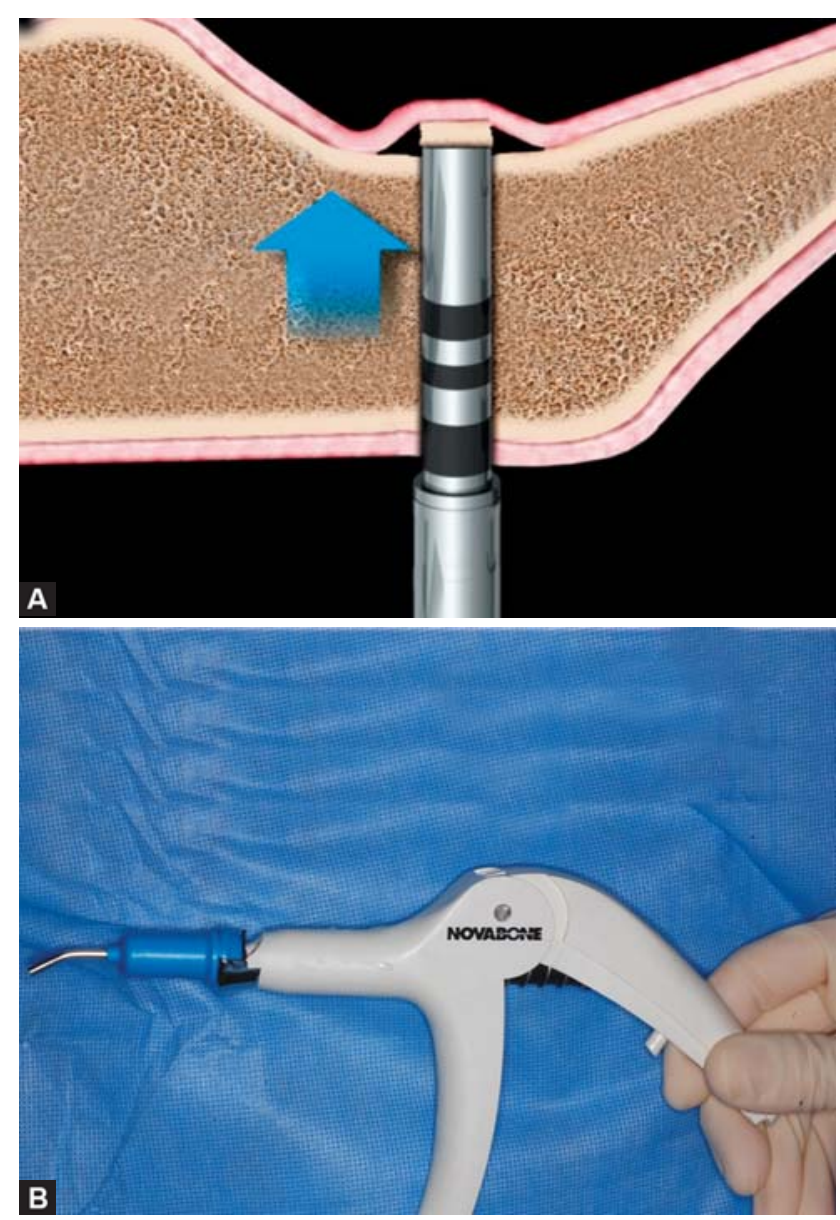

Figs 2A and B: The putty absorbs part of the forces that are applied to the bone and evenly distributors the remaining force while the risk of membrane perforation

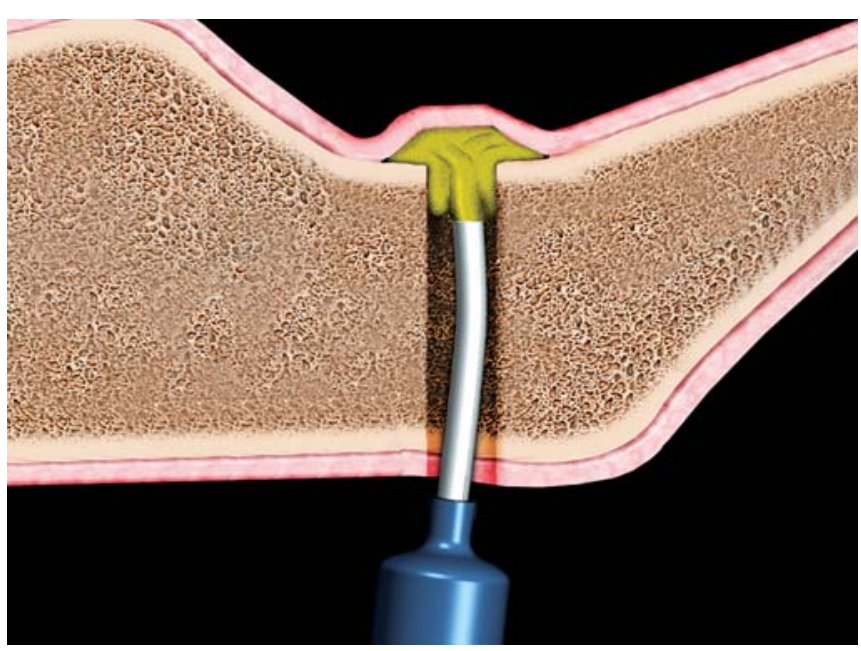

Fig. 3: The narrow tip of the delivery system allows it to enter the narrow osteotomy and reach the floor of the sinus 


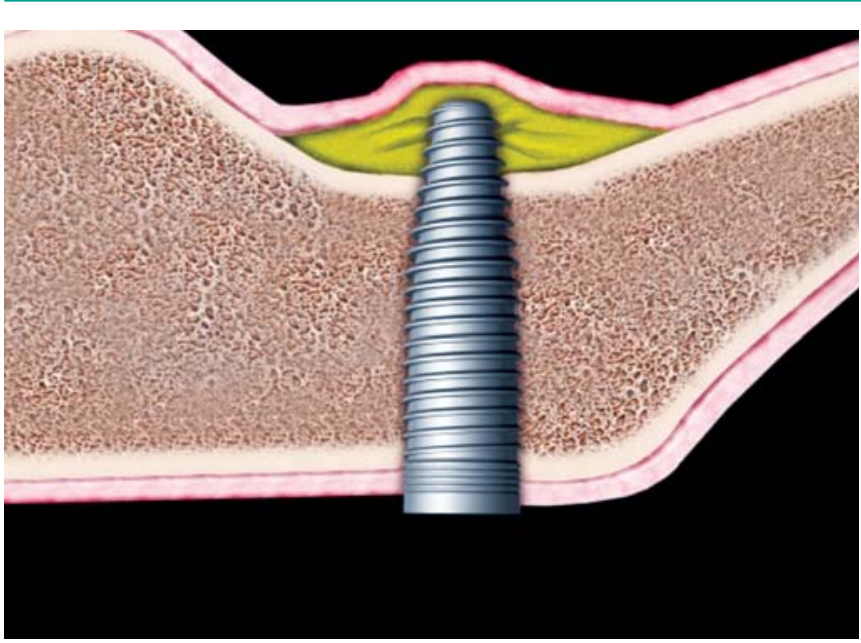

Fig. 4: The viscosity of the CPS putty that surrounds the apex of the implant aids in achieving increased primary stability

into the site. The hydraulic pressure from delivery of the graft material elevates the sinus membrane (Fig. 3). For every 0.5 cc injected into the sinus the floor is elevated approximately by $1 \mathrm{~mm}$.
Following adequate elevation of the sinus floor an implant is placed in the socket (Fig. 4). Human histomorphometric studies done in sockets have demonstrated that approximately $85 \%$ of the graft gets remodeled into vital bone in 5 to 7 months with approximately $15 \%$ residual graft after 6 months in the site. ${ }^{12}$

\section{Representative Case}

A 50 years old, healthy female (nonsmoker) presented for implant placement in the edentulous upper left premolar area. The subantral bone height was measured at $9.3 \mathrm{~mm}$ in the 24 area and $5.3 \mathrm{~mm}$ in the 25 area (Fig. 5A). The patient was premedicated with $2 \mathrm{gm}$ amoxicillin an hour before the surgery. Following local anesthesia initial drilling with a $2 \mathrm{~mm}$ twist drill, followed by a $2.9 \mathrm{~mm}$ drill to widen the osteotome to approximately $1.0 \mathrm{~mm}$ short of the sinus floor was performed utilizing a crestal approach (Neobiotech $\mathrm{SCA}^{\mathrm{TM}} \mathrm{kit}$ ). Subsequently, an appropriately sized (2.8 mm in 24 area and $3.2 \mathrm{~mm}$ in 25 area) S-reamer was utilized until the sinus floor was breached, while leaving the
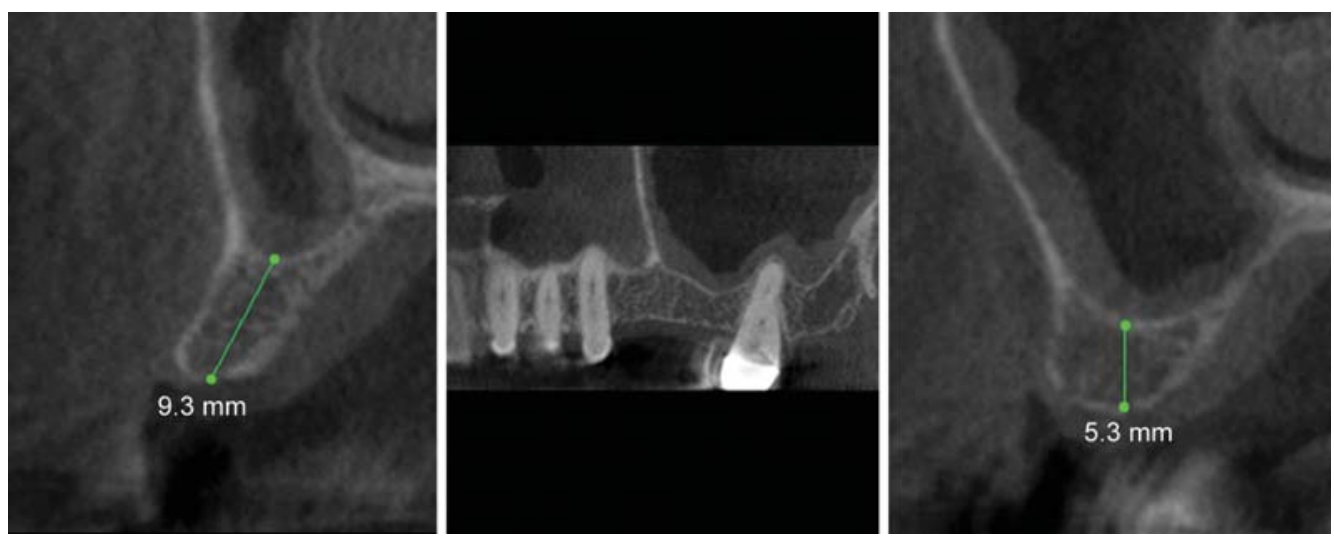

Fig. 5A: The cross-sections reveal the concave anatomy of the floor of the sinus. You can also note thickening of the sinus mucosa. Significant elevation of the floor of the sinus has to be performed in the area of the second premolar to allow for implant placement

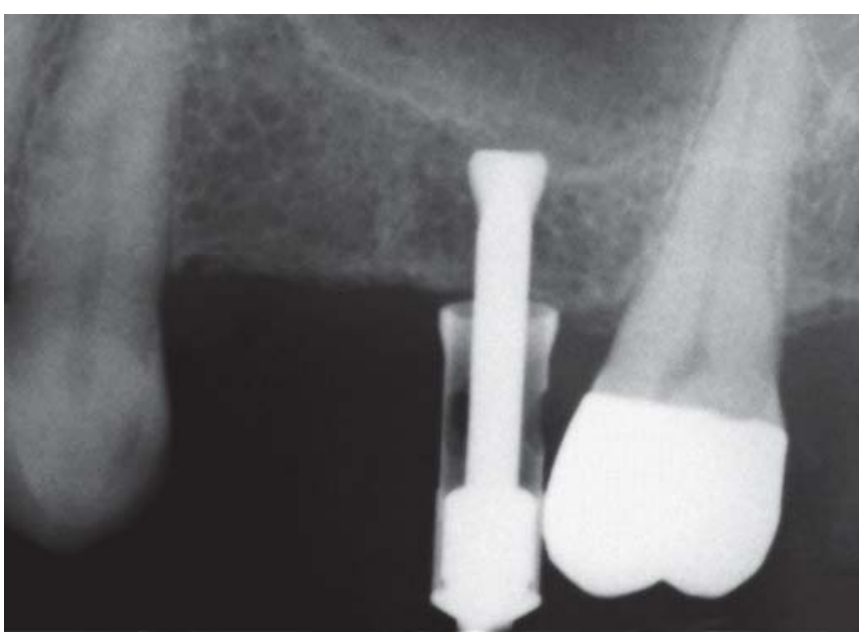

Fig. 5B: The reamer is specially designed to allow for removal of the bony floor of the sinus without perforating the Schneiderian membrane. Note: The loss of continuity of bone at the end of the reamer consistent with direct clinical contact of the reamer with the membrane

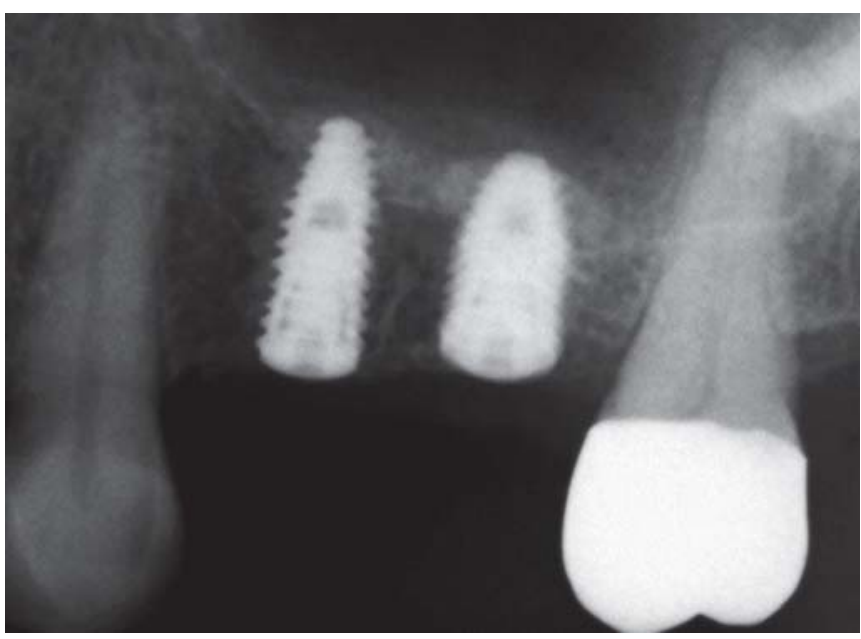

Fig. 5C: Immediate postoperative radiograph shows the sinus elevation achieved was adequate and both implants were successfully placed using this atraumatic technique 


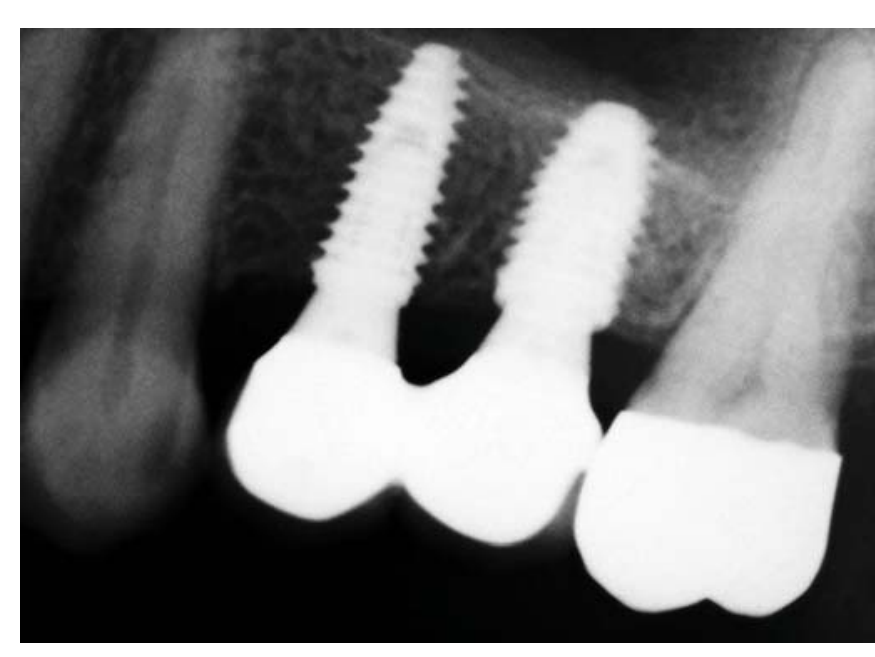

Fig. 5D: Seven-month postoperative radiograph demonstrates the trabecularization of the hard tissue at the apex of the implants, in the area of the sinus lift, is almost identical to the trabecularization of the native bone

membrane intact owing to the design of the reamer. Separation of the sinus floor was performed using a roundended depth gauge. Approximately, 0.5 cc CPS putty was injected into the \#24 area and 1.5 cc into the \#25 area (Figs 5B and C) using the cartridge delivery system and continued until the hydraulic pressure caused elevation of the sinus membrane. Once the membrane was adequately elevated as evidenced by the tactile sensation of resistance to additional bone grafting, the grafted material was laterally spread using a paddle-shaped bone spreader with a stopper running at $70 \mathrm{rpm}$. The $4 \times 10$ and $5 \times 8.5 \mathrm{~mm}$ CMI IS II implants were placed in \#24/25 areas, respectively. Implants were inserted with a primary stability greater than $35 \mathrm{~N} /$ $\mathrm{cm}^{2}$ in both sites and a healing abutment was placed for nonsubmerged healing. A 7-month postoperative radiograph demonstrated trabecular pattern in the grafted area indicative of the graft turnover and bone regeneration (Fig. 5D).

\section{DISCUSSION}

Lateral window approach offers a better control of the surgical site, particularly in a severely resorbed maxilla or when extensive implantation is needed. The approach using the Summers osteotomy was developed to simplify the sinuslift procedure using simultaneous sinus floor elevation and implantation in one stage through the socket. However, both approaches seem to yield similar success rates. ${ }^{13-16}$

A less invasive and more predictable TSFE technique was conceived by exploiting the superior handling characteristics and unique delivery system of CPS putty. The consistency of CPS putty also helps in minimizing membrane perforations and associated adverse events. The technique is also an attempt to reduce the grafting to the minimum while generating adequate bone volume required for optimal osseointegration and anchorage of the implants. CPS putty in unidose cartridges facilitates precise delivery of the graft material and controlled elevation of the sinus membrane. Additional advantages of this technique are reduced chairside times and minimal graft wastage.

CPS putty in the cartridge system for sinus floor elevation offers a more conservative procedure, localized augmentation of sinus and less postoperative morbidity. This technique can be successfully used for sinus augmentation with immediate implant placement, as it offers key primary stability to the implant. All these advantages make TSFE when used in conjunction with CPS putty a viable option for implant placement in the posterior maxilla.

\section{CONCLUSION}

This article introduces a novel technique for indirect sinus elevation. The use of CPS putty for indirect sinus lift provides a clinically safe and effective option for simultaneous placement of implants that allows for a less invasive approach, less complications and minimum discomfort for the patient.

\section{REFERENCES}

1. Summers RB. A new concept in maxillary implant surgery: the osteotome technique. Compendium 1994 Feb;15(2):152-162.

2. Hernández-Alfaro F, Torradeflot MM, Marti C. Prevalence and management of Schneiderian membrane perforations during sinus-lift procedures. Clin Oral Implants Res 2008 Jan;19(1): 91-98.

3. Engelke W, Deckwer I. Endoscopically controlled sinus floor augmentation. A preliminary report. Clin Oral Implants Res 1997 Dec;8(6):527-531.

4. Kfir E, Goldstein M, Yerushalmi I, Rafaelov R, Mazor Z, Kfir $\mathrm{V}$, Kaluski E. Minimally invasive antral membrane balloon elevation - results of a multicenter registry. Clin Implant Dent Relat Res 2009 Oct;11(Suppl 1):e83-e91.

5. Ahn SH, Park EJ, Kim ES. Reamer-mediated transalveolar sinus floor elevation without osteotome and simultaneous implant placement in the maxillary molar area: clinical outcomes of 391 implants in 380 patients. Clin Oral Implants Res 2012;23(7): 866-872.

6. Aloy-Prósper A, Maestre-Ferrin L, Peñarrocha-Oltra D, Peñarrocha-Diago M. Bone regeneration using particulate grafts: an update. Med Oral Patol Oral Cir Bucal 2011 Mar;16(2): e210-e214.

7. Rickert D, Slater JJ, Meijer HJ, Vissink A, Raghoebar GM. Maxillary sinus lift with solely autogenous bone compared to a combination of autogenous bone and growth factors or (solely) bone substitutes. A systematic review. Int J Oral Maxillofac Surg 2012 Feb;41(2):160-167.

8. Kotsakis G, Chrepa V, Marcou N, Prasad H, Hinrichs J. Flapless alveolar ridge preservation utilizing the 'socket-plug' technique: clinical technique and review of the literature. J Oral Implantol 2012 Nov 12. [Epub ahead of print]

9. Kotsakis G, Chrepa V, Katta S. Practical application of the newly introduced natural bone regeneration (NBR) concept utilizing 
alloplastic putty. Int J Oral Implantol Clin Res 2011 SepDec;2(3):145-149.

10. Xynos ID, Edgar AI, Buttery LDK, Hench LL, Polak JM. Geneexpression profiling of human osteoblasts following treatment with the ionic products of Bioglass 45S5 dissolution. J Biomed Mater Res 2001 May;55(2):151-157.

11. Mahesh L, Salama MA, Kurtzman GM, Joachim FP. Socket grafting with calcium phosphosilicate alloplast putty: a histomorphometric evaluation. Compend Contin Educ Dent 2012 Sep;33(8):e109-e115.

12. Lanka M, Kotsakis G, Venkataraman N, Shukla S, Prasad H. Ridge preservation with the socket-plug technique utilizing an alloplastic putty bone substitute or a particulate xenograft: a histological pilot study. J Oral Implantol 2013 Jun 17 [Epub ahead of print].

13. Peleg M, Garg AK, Mazor Z. Predictability of simultaneous implant placement in the severely atrophic posterior maxilla: a 9-year longitudinal experience study of 2132 implants placed into 731 human sinus grafts. Int J Oral Maxillofac Implants 2006 Jan-Feb;21(1):94-102.

14. Peleg M, Mazor Z, Chaushu G, Garg AK. Sinus floor augmentation with simultaneous implant placement in the severely atrophic maxilla. J Periodontol 1998 Dec;69(12): 1397-1403.

15. Peleg M, Mazor Z, Garg AK. Augmentation grafting of the maxillary sinus and simultaneous implant placement in patients with 3 to $5 \mathrm{~mm}$ of residual alveolar bone height. Int J Oral Maxillofac Implants 1999 Jul-Aug;14(4):549-556.

16. Fermergard R, Astrand P. Osteotome sinus floor elevation and simultaneous placement of implants. A 1-year retrospective study with Astra Tech implants. Clin Implant Dent Relat Res 2008 Mar;10(1):62-69.

\section{ABOUT THE AUTHORS}

\section{Ziv Mazor}

Private Practice, Ra'anana, Israel

\section{Andreas loannou}

Resident, Department of Advanced Education in Periodontology Division of Periodontology, University of Minnesota, Minneapolis Minnesota, United States

\section{Narayan Venkataraman}

Private Practice, Bengaluru, Karnataka, India

\section{George Kotsakis (Corresponding Author)}

Resident, Department of Advanced Education in Periodontology, Division of Periodontology, University of Minnesota, Minneapolis, Minnesota United States, Phone: (651) 395-9200, e-mail: geo.kotsakis@gmail.com 